\title{
Etat Des Lieux Et Gestion Des Semences Des Principales Céréales Cultivées Au Nord De La Côte d'Ivoire
}

\author{
Kouablan Edmond Koffi \\ Centre National de Recherche Agronomique (CNRA), \\ Laboratoire Centrale de Biotechnologie (LCB), Côte d'Ivoire \\ Saraka Didier Martial Yao \\ Nafan Diarrassouba \\ Laopé Ambroise Casimir Siene \\ Université Peleforo Gon Coulibaly (UPGC) \\ UFR des Sciences Biologiques, Korhogo, Côte d'Ivoire
}

doi: 10.19044/esj.2016.v13n3p112 URL:http://dx.doi.org/10.19044/esj.2016.v13n3p112

\begin{abstract}
The survey aims at assessing current managing status of organizations involves in the conservation of local genetic diversity of rice, maize, sorghum and pearl millet in seedbanks located in northern Côte d'Ivoire. An investigation was conducted with 100 respondents in charge of cereal seedbanks managing from Ferkessedougou and Ouangolodougou areas. The results at regional scale revealed two co-existing informal and formal systems for seedbanks management in these areas. The farmers' seedbanks system or informal system was the main source of cereal seed supply for farmers and more important than the national or formal seedbank system. Only, farmers' seedbanks of two crops, rice and maize were found in visited localities. The management of these farmers' seedbanks was enabled by associations or cooperatives gathering more men (78.27\%) than women (21.73\%). Ninety percent (90\%) of respondents were identified as saving 1 to 3 traditional varieties of cereal in these seedbanks managed by farmers in the studied region. The reveled difficulties in the farmers' seedbanks management appeared mainly as informally organized seed system that must be a complementary approach to formal system or national seed system for genetic diversity preservation of local cereal varieties and also as a distribution channel of improved cereal varieties.
\end{abstract}

Keywords: Rice, maize, sorghum, pearl millet, farmers’ seedbank, Côte d'Ivoire 


\section{Résumé}

Dans l'objectif d'optimiser la conservation des ressources phytogénétiques des principales céréales produites dans le Nord de la Côte d’Ivoire, la présente étude fait l'état des lieux et l'inventaire des structures impliquées dans la gestion des semences de riz, de maïs, de sorgho et de mil. La méthodologie repose sur une enquête réalisée auprès de 100 personnes en charge de la gestion des banques de semences dans les localités de Ferkessédougou et de Ouangolodougou. Les enquêtes ont révélé la coexistence de deux systèmes semenciers que sont le système semencier paysans ou informel à travers les banques communautaires et le système étatique ou formel. Seulement, la présence de banques communautaires a été observée dans le cas de semences de riz et de maïs, dans ces localités. La gestion de ces banques communautaires est assurée sous formes d'associations ou de coopératives regroupant une proportion élevée d'hommes (78,27\%) que de femmes (21,73\%). Quatre-vingt dix pour cent (90\%) des répondants ont identifié 1 à 3 variétés de céréales gérées dans les banques communautaires de la région. Des difficultés dans la gestion de ces banques communautaires de semences ont été rapportées par les paysans. Cela démontre que le système semencier paysans ou informel doit être un complément du système semencier national pour la préservation des variétés locales et comme canal de diffusion des variétés améliorées de céréales.

Mots-clés: Riz, maïs, sorgho, mil, banques communautaires de semences, Côte d'Ivoire

\section{Introduction}

Les céréales constituent la base de l'alimentation pour la majorité des populations, qu'elles soient consommées en grains, en farine ou en bouillie. En 2015 la production mondiale de céréales était estimée à 2047 million de tonnes (CIC, 2016). En Côte d'Ivoire, les céréales ainsi que les plantes à racines, les tubercules, les protéagineux, les espèces maraîchères et les fruitiers, constituent les cultures de base, indispensables à la sécurité alimentaire (Sangaré et al., 2009). En 2015, la production nationale céréalière a représenté $12,3 \%$ du niveau national de production des cultures vivrières estimé à 10,7 millions de tonne. Parmi ces céréales, la production du riz a été de 684800 t et celle de maïs, de mil et de sorgho estimée ensemble à $631300 \mathrm{t}$ (DGPE, 2015). Contrairement au riz et au maïs qui sont cultivés dans toutes les régions agro-écologiques de la Côte d'Ivoire, le mil et le sorgho sont traditionnellement cultivés dans la région du Nord entre le $8^{\mathrm{e}}$ et le $11^{\mathrm{e}}$ degré de latitude Nord. Ces céréales (riz, maïs, mil et sorgho) sont produites pour les besoins alimentaires des ménages ainsi que ceux des 
marchés locaux et internationaux. De plus, les résidus de récolte de céréales sont aussi utilisés comme source importante de fourrage pour l'alimentation des animaux d'élevage, l'une des principales activités dans le Nord de la Côte d'Ivoire.

La communauté scientifique internationale a officiellement reconnu l'importance des contributions des populations locales et surtout des agriculteurs dans la conservation des ressources génétiques pour le bien-être des populations présentes et futures (OUA, 2002). En effet, les agriculteurs interviennent dans la mise en place et la conservation des ressources phytogénétiques comme le souligne l'article 9 du traité international des ressources phytogénétiques (FAO, 2009). En dépit de plusieurs initiatives de développement du secteur des semences tant au niveau mondial que national, les agriculteurs ont accès difficilement aux semences certifiées ou améliorées. La plupart des semences détenues par les paysans proviennent de sources informelles de production, soit de trocs entre agriculteurs locaux ou d'achats sur les marchés locaux, urbains et périurbains. En termes de production, le paysan intervient dans la sélection des variétés et semences les mieux adaptées à son environnement. Il pratique l'autoproduction des semences en choisissant chaque année les meilleures semences. Le système semencier informel est basé également sur la valorisation des connaissances endogènes et fournit environ $70 \%$ des semences de céréales et autres cultures vivrières. Ainsi, la diversité des ressources phytogénétiques et leur production à l'échelle locale semble influencée par la gestion des semences détenues par les paysans. Or, aucune étude assez poussée n'a été menée en Côte d'Ivoire pour faire l'état de la conservation en champ de la biodiversité de ces ressources phytogénétiques utiles à l'alimentation et à l'agriculture ainsi que de leur gestion dans les banques de semences communautaires. L'absence de telles informations est préjudiciable à la préservation de la diversité des ressources phytogénétiques, à l'instauration d'une agriculture durable et à la sécurité alimentaire en Côte d’Ivoire. La présente étude s'inscrit, donc, dans le cadre de la conservation des semences des principales céréales cultivées dans le Nord de la Côte d'Ivoire que sont le riz, le maïs, le sorgho et le mil. Cette étude vise principalement à faire l'état des lieux et l'inventaire des structures de gestion des semences de ces quatre céréales dans les localités de Ferkessédougou et Ouangolodougou situées au Nord de la Côte d'Ivoire.

\section{Matériel et méthodes}

\section{Zone d'étude}

L'étude a été conduite au Nord de la Côte d'Ivoire dans les localités de Ferkessédougou et de Ouangolodougou appartenant à la région du Tchologo qui s’étend sur une superficie de 17043 km² (Figure 1). La région 
du Tchologo est située dans la partie septentrionale de la Côte d'Ivoire caractérisée par un climat de type soudano-guinéen avec deux grandes saisons : une sèche et une humide. La pluviométrie moyenne annuelle varie entre $900 \mathrm{~mm}$ et $1400 \mathrm{~mm}$, avec une température moyenne annuelle variant entre $27{ }^{\circ} \mathrm{C}$ et $28{ }^{\circ} \mathrm{C}$. La végétation de type savanicole est constituée de savane arborée et de forêt clairsemée propice à l'élevage des herbivores. A l'image des régions situées dans la zone soudano-guinéenne de la Côte d'Ivoire, l'agriculture, essentiellement sous pluie, et l'élevage extensif constituent les principales activités de la région du Tchologo (Silue et al., 2014).

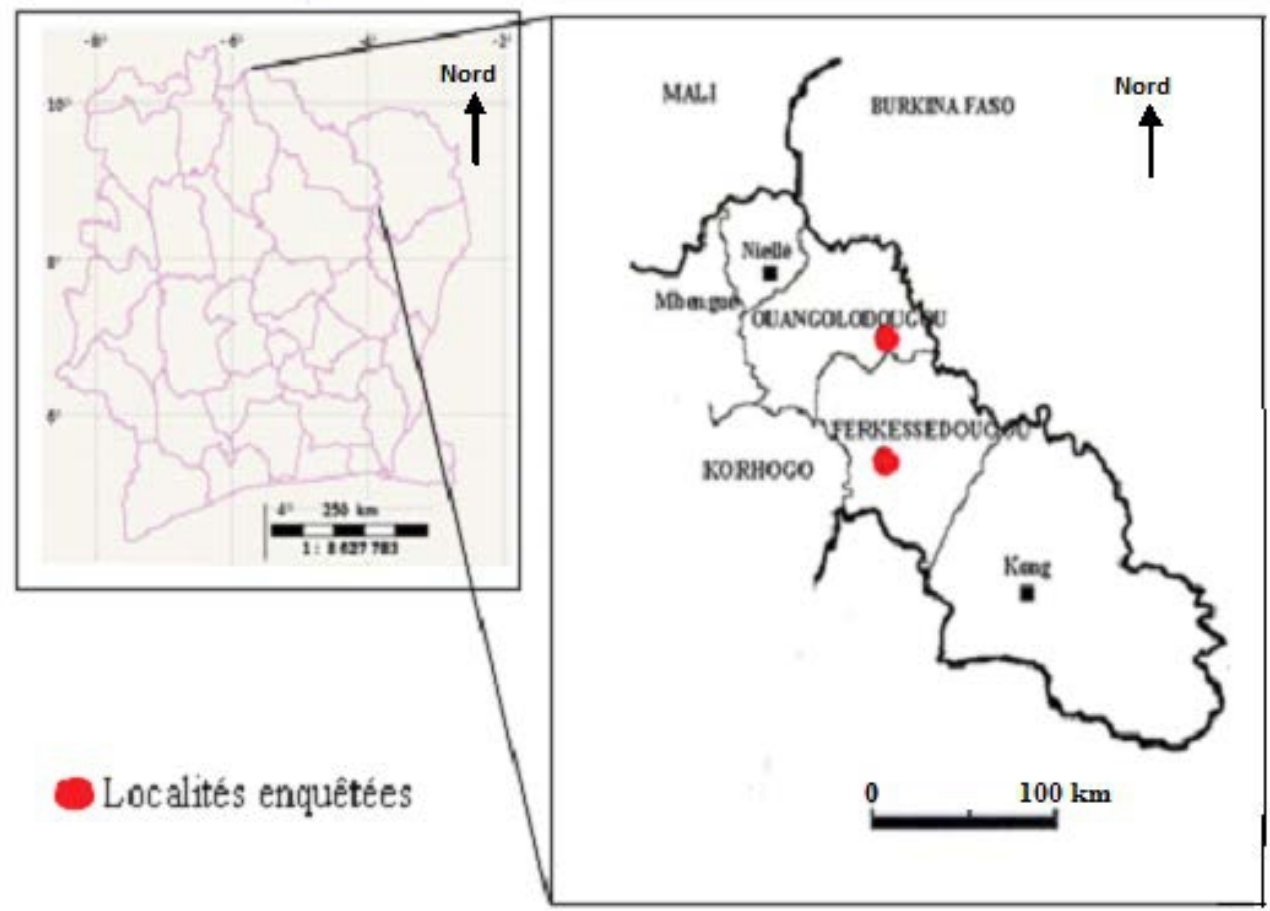

Figure 1. Carte de la région du Tchologo indiquant les localités enquêtées de Ferkessédougou et de Ouangolodougou, au Nord de la Côte d'Ivoire

\section{Conduite de l'enquête}

L'enquête a été conduite dans 6 villages des localités de Ferkessédougou et de Ouangolodougou (Tableau 1). Au cours de l'enquête, des cultivateurs et des représentants des structures de gestion de semences de riz, maïs, mil et sorgho ont été interrogés. Un effectif de 100 répondants pour l'ensemble des 6 villages a été soumis à un questionnaire au cours d'une enquête effectuée en décembre 2015, selon les outils et techniques d'une approche de recherche participative basée sur des observations directes, des discussions libres et des entretiens individuels. Alors que le choix des villages visités a été fait selon le programme de travail des agents de 
l'Agence National d'Appui pour le Développement Rural (ANADER), celui des personnes interrogées a été aléatoire. Dans les villages, les entretiens ont été conduits avec l'aide des traducteurs locaux et les autorités locales telles que les Chefs de village ou notables ou délégués pour faciliter les échanges. Les informations ont porté sur, (i) la structure de gestion de semences de céréale, (ii) les modes de gestion de la banque de semence de céréale et (iii) les systèmes de production et d'approvisionnement de semences de céréales.

\section{Traitements statistiques des données de l'enquête}

Le logiciel Excel version 2007 à servi à la fois à la saisie des données brutes de l'enquête et à leur analyse. L'analyse a consisté aux calculs de certains paramètres de statistiques descriptives (fréquence, pourcentage de réponses, moyenne) et à l'élaboration de graphiques (à barres et à secteurs).

\section{Résultats}

\section{Structures de gestion de semences de céréales rencontrées}

Caractéristiques générales des structures de gestion de semences de céréales

Un effectif de huit (8) structures de gestion de semences de céréales a été visité dont trois (3) à Ferkessédougou (Association des semenciers du Tchologo, CNRA et ANADER) et 5 à Ouangolodougou (Association solution, Bèguéléman, Binkadi, Chigata et Onimeta). Parmi ces structures, six (6) sont des banques communautaires de semences (Association des Semenciers du Tchologo, Association Solution, Bèguéléman, Binkadi, Chigata et Onimeta) et 2 sont des structures étatiques (CNRA et ANADER) (Tableau 1). Les structures étatiques que sont le CNRA et l'ANADER ont en charge la gestion de semences traditionnelles et améliorées de riz, maïs, sorgho et mil.

Sur la totalité des 100 personnes interrogées dans les deux localités étudiées 78,27 \% d'hommes et 21,73\% de femmes ont été impliqués dans la gestion des banques de semences de céréales. Les femmes ont été impliquées seulement dans la gestion des banques de semences de riz $(17,4 \%)$ et maïs (4,34 \%) dans les localités prospectées (Figure 2). 
Tableau 1. Liste des structures de gestion de semences de riz, maïs, de sorgho et de mil dans les localités de Ferkessédougou et de Ouangolodougou, au Nord de la Côte d’Ivoire.

\begin{tabular}{|c|c|}
\hline Localités / Villages & Structures de gestion de semences \\
\hline Ferkéssédougou & Association des semenciers du Tchologo ${ }^{a}$ \\
\hline Ferkéssédougou & $\mathrm{CNRA}^{\mathrm{b1}}$ \\
\hline Ferkéssédougou / Sambakaha & ANADER $^{\text {b2 }}$ \\
\hline Ouangolodougou / Nieronigué & Association solution $^{\mathrm{a}}$ \\
\hline Ouangolodougou / Noumoussokpassola & Bèguéléman $^{\mathrm{a}}$ \\
\hline Ouangolodougou / Niangbarasso & Binkadi $^{\mathrm{a}}$ \\
\hline Ouangolodougou / Kafokaha & Chigata $^{\mathrm{a}}$ \\
\hline Ouangolodougou / Bèhkaha & Onimeta $^{\mathrm{a}}$ \\
\hline
\end{tabular}

${ }^{1}$ CNRA : Centre National de Recherche Agronomique

${ }^{2}$ ANADER : Agence Nationale pour le Développement Rural

${ }^{\mathrm{b}}$ Structures étatiques de gestion des semences (système semencier formel)

${ }^{a}$ Structures non étatiques ou banques communautaires de gestion des semences (système semencier informel)

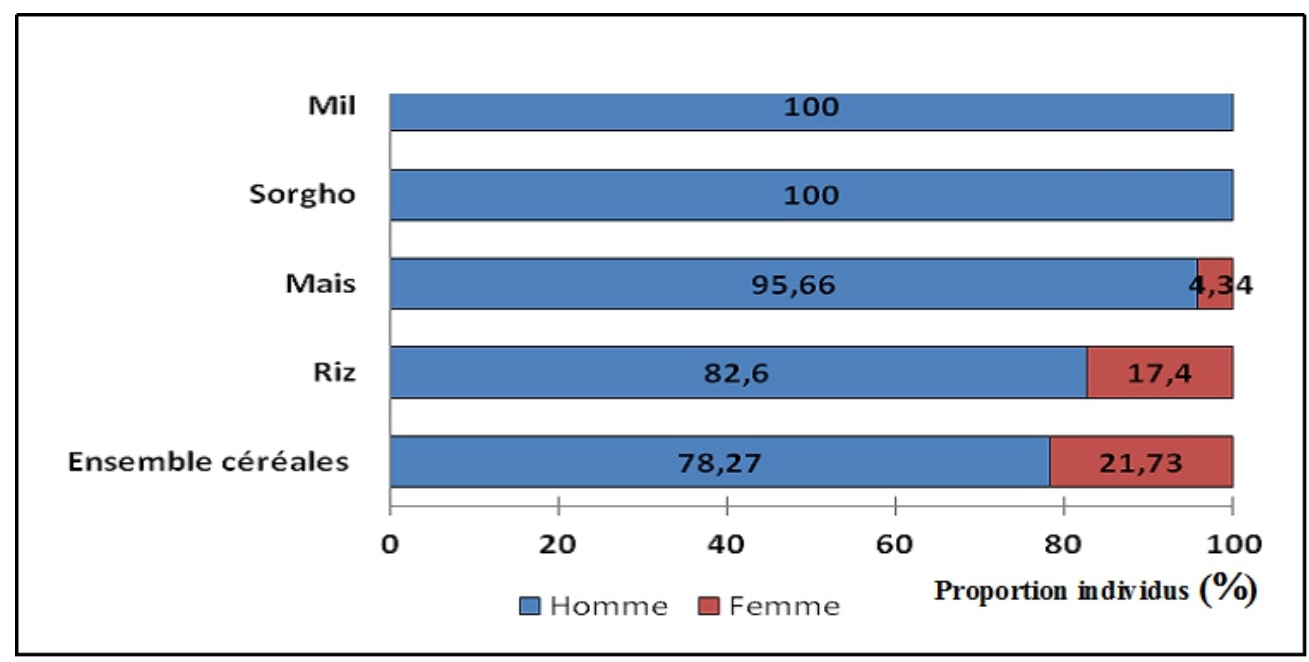

Figure 2. Proportions d'individus classés par genre impliqués dans la gestion des banques de semences dans les localités de Ferkessédougou et de Ouangolodougou, au Nord de la Côte d'Ivoire.

\section{Caractéristiques des banques communautaires de semences de céréales}

Les activités des six (6) banques communautaires de semences identifiées dans les localités étudiées ont porté sur la conservation des semences de riz et de maïs. Aucune banque communautaire de sorgho, ni de mil n'a été identifiée dans les localités de Ferkessédougou et Ouangolodougou. A partir des informations recueillies auprès des gestionnaires des banques communautaires de semences de céréales identifiées à Ferkessédougou et à Ouangolodougou, les productions moyennes de 2011 à 2015 de semences de riz ont varié entre 0,36 t (Chigata et Binkadi) et 2,91 $\mathrm{t}$ (Association des semenciers du Tchologo). Sur la même période, la banque communautaire dénommée Onimeta a produit respectivement 0,69 t et 0,54 $t$ de semences de maïs et de riz (Figure 3). 


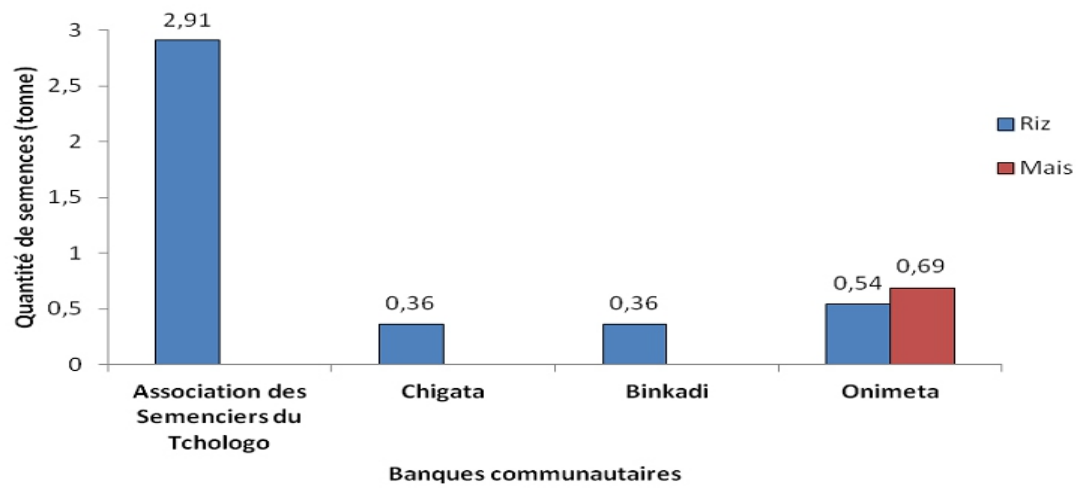

Figure 3. Quantités moyennes de semences de riz et de maïs produites dans quatre des six banques communautaires à Ferkessédougou et à Ouangolodougou, au Nord de la Côte d'Ivoire.

A la question de savoir le nombre de variétés de céréales connues, 10 $\%$ des répondants ont rapporté n’ayant aucune connaissance des variétés de riz, de maïs, de mil et de sorgho cultivées dans la région. A partir des $90 \%$ des personnes interrogées ayant une idée des caractères distinctifs des céréales, le nombre de variétés connues de riz (1 à 3 variétés) rapporté par 2 à $26 \%$ des répondants a été plus important que celui de maïs et de mil (1 à 2 variétés) ainsi que de Sorgho (1 variété) rapportés par 2 à $14 \%$ des répondants (Figure 4).

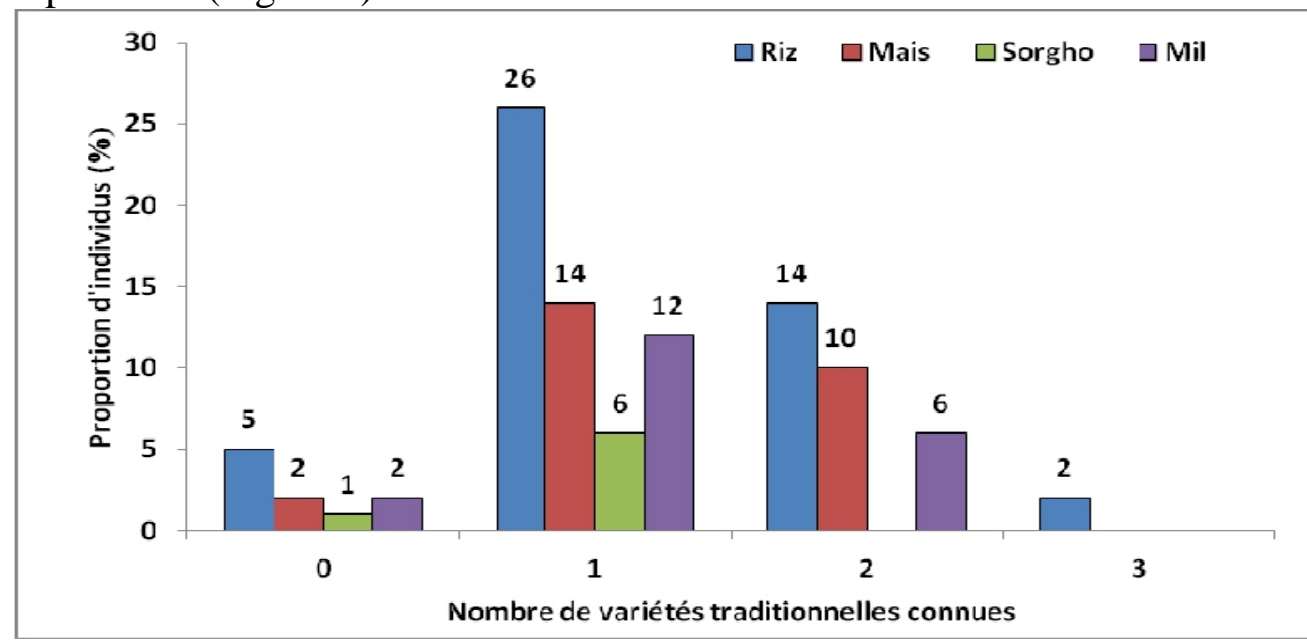

$0=$ Aucune variété de céréale identifiée par les répondants

1 ou 2 ou 3 = nombre de variété de céréale identifiée par les répondants.

Figure 4. Nombres de variétés de riz, maïs, sorgho et mil connues et rapportées par les personnes impliquées dans la gestion des banques communautaires recensées à

Ferkessédougou et à Ouangolodougou, au Nord de la Côte d’Ivoire. 


\section{Gestion des banques de semences de céréale}

Les banques de semences communautaires identifiées sont détenues par des associations ou des coopératives de producteurs qui sont régies par des statuts et règlements intérieurs et par des règles établies par la communauté. Aucun réseau n'existe entre ces banques communautaires. Les différentes banques communautaires de semences de riz identifiées ont été essentiellement approvisionnées par Africarice. La gestion des banques de semences de maïs, de mil et de sorgho du CNRA à Ferkessédougou est directement rattachée au Département des Ressources Génétiques du CNRA à Abidjan. La plupart des banques communautaires ne bénéficient d'aucun soutien financier et technique pour la conservation des semences. Lorsque ces banques communautaires bénéficient d'un appui, celui-ci est relatif à la fourniture de semences de variétés améliorées et des engrais, en vue de les multiplier pour constituer la semence de base et le renforcement de leurs capacités techniques.

\section{Systèmes de production et d'approvisionnement de semences Systèmes de production de semences}

L'enquête a révélé deux systèmes de production de semences. La première est relative au mode de production par les paysans sur la base d'observation suivie de la sélection des meilleurs épis sur les plants au champ. Les choix se font suivant les caractères morphologiques tels que la robustesse des pieds, la précocité de floraison, la résistance aux ravageurs et le rendement élevé en grains (Figure 5). A côté de ce mode de production de semences, existe une structure plus spécialisée dans la multiplication et la vente des semences de riz (Association des Semenciers du Tchologo) identifiée à Ferkessédougou. L’Association des Semenciers du Tchologo ne bénéficiant pas de suivi appuyé des structures étatiques produit les semences de riz selon un système non encore conventionnel. 

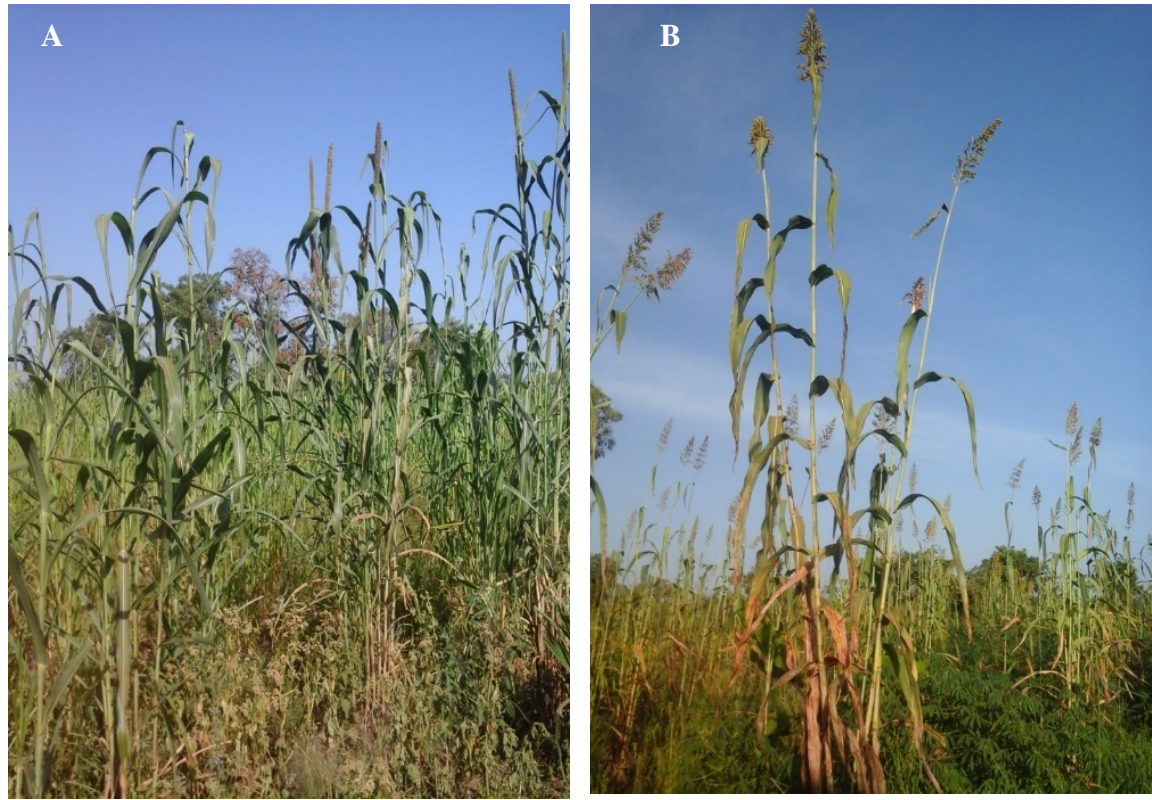

Figure 5. Stades de développement au champ de pieds de mil en phase d'épiaison (A) et de sorgho en phase de maturation (B) favorables à la sélection paysanne des semences à conserver pour la prochaine culture.

\section{Systèmes d'approvisionnement en semences}

Les résultats ont montré que $49 \%$ des paysans s'autoapprovisionnent en semences à partir de leurs productions. Une proportion de $20 \%$ des paysans s'approvisionne par le système de trocs. Les autres paysans s'approvisionnent soit dans les banques de semences (16\%), soit sur les marchés (15 \%) urbains et périurbains de vente de semences (Figure 6).

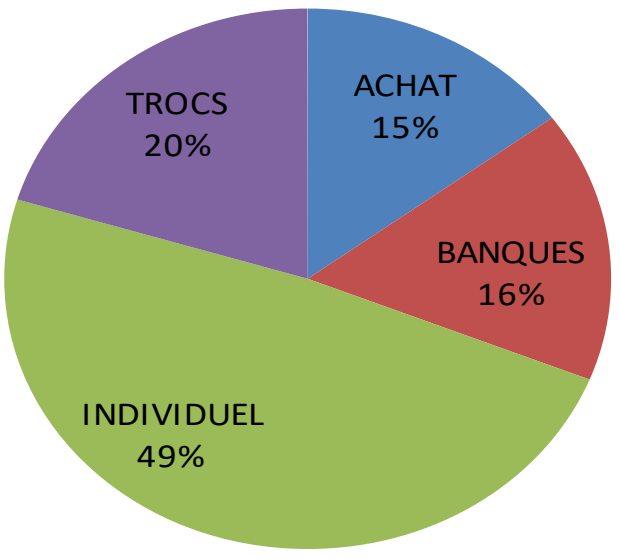

Figure 6. Proportions des différents modes d’approvisionnement des paysans en semences dans les localités de Ferkessédougou et de Ouangolodougou, au Nord de la Côte d’Ivoire. 


\section{Discussion}

Le système semencier a pour rôle de fournir aux cultivateurs, en temps opportun, des semences de qualité et en quantité suffisantes (Coulibaly et al., 2008). Cette étude a mis en évidence deux systèmes semenciers dans la région du Tchologo couvrant les localités de Ferkessédougou et Ouangolodougou. Il s'agit du système semencier paysan ou les banques de semences communautaires informelles gérées localement par les paysans eux-mêmes et du système semencier étatique formel géré par des structures nationales telles que l'Agence Nationale d'Appui pour le Développement Rurale (ANADER) et le Centre National de Recherche Agronomique (CNRA). L'étude a révélé que dans la région du Tchologo, le système semencier paysan, informel, est le principal fournisseur de semences pour le paysan, loin devant le système semencier formel ou étatique. Ces résultats doublés par le constat de l'accès difficile des paysans aux semences améliorées de céréales, constaté lors de l'enquête, traduisent ensemble la méconnaissance des circuits d'approvisionnement de ces semences de qualité créées par la recherche (CNRA) et diffusées par l'ANADER. Des résultats similaires ont été rapportés par Coulibaly et al. (2008) dans l'étude des systèmes d'approvisionnement en semences de mil et sorgho au Mali, pays frontalier au Nord de la Côte d'Ivoire.

Outre les structures de gestion étatique (ANADER et CNRA), la gestion des banques communautaires de semences des céréales étudiées dans le Nord de la Côte d'Ivoire est en général attribuée aux hommes conformément à la structuration des sociétés traditionnelles africaines où l'homme est le chef de famille. En effet, contrairement aux hommes, les rôles dévolus aux femmes dans les ménages sont essentiellement l'utilisation des semences pour la culture, la préparation de la nourriture quotidienne de la famille, la production et la vente de mets confectionnés à base de céréales et l'éducation des enfants. Des rôles similaires de la femme dans les sociétés africaines ont été rapportés en Côte d'Ivoire par Assa et al. (2006), Assa et al. (2009) et Djeni (2009). Cela explique la faible implication des femmes dans la gestion des banques communautaires de céréales à Ferkessédougou et Ouangolodougou.

Les résultats ont révélé l'inexistence de banques de gènes communautaires de mil et de sorgho dans les localités étudiées. Les semences des variétés traditionnelles de mil et de sorgho sont détenues par des paysans individuellement qui les échangent entre eux. Le fait que les échanges restent gratuits en milieu paysan constitue un handicap pour la diffusion des semences des variétés améliorées de ces deux espèces (Beninga, 2007 ; Coulibaly et al., 2008). En effet, pour ces deux céréales, en plus des variétés traditionnelles, il existe quelques variétés améliorées produites par la recherche, dont le CNRA en particulier et diffusées par 
l’ANADER (Sangaré et al., 2009). Ces variétés améliorées sont plus productives et adaptées aux changements climatiques.

Les détenteurs de banques de gènes communautaires ont généralement recours aux variétés améliorées fournies principalement lors d'exécution de projets. En effet, l'ANADER, à travers le Projet de Réhabilitation Agricole et Réduction de la Pauvreté (PRAREP), avait mis à la disposition des paysans des semences améliorées de riz et de maïs. Cela explique la disparition progressive d'un grand nombre des variétés traditionnelles de riz et de maïs dans la région ainsi que la faible diversité des variétés enregistrées dans les banques communautaires. Aussi, la non sensibilisation des paysans sur l'importance des variétés traditionnelles comme source indispensable de création de nouvelles variétés pour faire face aux nouveaux défis environnementaux expliquerait le faible nombre de variétés traditionnelles connues dans la région.

Les banques de semences communautaires qui ont été identifiées dans les localités de Ferkessédougou et de Ouangolodougou rencontrent d'énormes difficultés dans leur gestion et dans leur mode de fonctionnement. En plus, les banques de gènes communautaires souffrent de l'absence de soutiens financier et technique des structures d'appui pour la conservation et l'utilisation durable du matériel végétal détenu. Il n'existe pas de programmes spécifiques de recherche et de développement visant la conservation des variétés traditionnelles dans des infrastructures de conservation appropriées. Il convient de noter que le domaine des ressources génétiques souffre de l'absence d'un cadre réglementaire approprié (inexistence actuellement de textes règlementaires nationaux sur les ressources phytogénétiques) et d'un cadre institutionnel approprié (inexistence d'une structure formelle chargée de la gestion des ressources phytogénétiques). Un certain nombre de difficultés ont été relevées dans la mise en place, la gestion et l'entretien des banques de semences communautaires. Il s'agit entre autres des difficultés liées à l'abandon des variétés traditionnelles, au manque de formation des paysans aux activités de récoltes et de post-récoltes (conditionnement, stockage et conservation), à la méconnaissance des textes existants sur les semences par les détenteurs des banques et à l'inexistence de réseau d'échanges entre les banques de gènes elles-mêmes et les autres initiatives locales ou régionales (Sangaré et al., 2009).

Les systèmes paysans de production et d'approvisionnement de semences (sélection, échanges et introductions de variétés, etc.) décrites dans la présente étude sont des pratiques largement connues et déjà rapportées sur diverses spéculations dans la sous-région Ouest-africaine (Dansi et al., 2010; Barro-Kondombo et al., 2010 ; Missihoun et al., 2012). Pour la constitution de banques individuelles ou communautaires de gènes de riz, maïs, mil et 
sorgho dans la région du Tchologo, la sélection de semences repose sur les savoirs endogènes locaux. Les agriculteurs pratiquent une sélection massale ou phénotypique à chaque génération pendant les récoltes en fonction d’objectifs de production et une appréciation propre des variétés (Coulibaly et al., 2008). La régénération des stocks de semences chaque année en milieu paysan conduit à une évolution adaptative des variétés. Vu dans ce sens les variétés entretenues par le système paysan sont adaptées à l'agro-climat local mais sont peu productives comparativement aux variétés améliorées diffusées par la recherche. Ainsi, le système semencier paysan apparaît comme un complément essentiel du système semencier national pour la préservation des variétés locales et comme canal de diffusion des variétés améliorées (Bazile et Abrami, 2008).

\section{Conclusion}

Les enquêtes réalisées sur l'état des lieux et les structures de gestion des semences des principales céréales cultivées ont permis de caractériser le système semencier de la région du Tchologo. Il ressort que deux systèmes semenciers coexistent dans la région. Il s’agit du système semencier paysan ou informel plus prépondérant et du système semencier étatique ou formel. Contrairement au riz et au maïs, l'inexistence de banque de gènes communautaires de mil et de sorgho dans les différentes localités visitées a été constatée. Les banques de gènes communautaires de riz et maïs identifiées sont gérées par des associations ou coopératives regroupant une proportion élevée d'hommes que de femmes. Les paysans s’approvisionnent en semences à partir de leurs propres productions ou par troc ou dans les structures productrices de semences. Par ailleurs, à l'image des localités de Ferkessédougou et de Ouangolodougou des défis majeurs restent encore à relever, afin de faire interagir davantage les systèmes semenciers paysans et formels pour assurer une agriculture et une sécurité alimentaire plus durables en Côte d'Ivoire.

\section{References:}

1. Assa, R., Konan, J.L., Nemlin, J., Prades, A., Agbo, N., \& Sie, R.S. (2006). Diagnostic de la cocoteraie paysanne du littoral ivoirien. Sciences \& Nature, 3 (2) :113-120.

2. Assa, R., Konan, J.L., \& Bonny, C. (2009). Etat des lieux de la filière cocotier en Côte d'Ivoire : évaluation des besoins d'appui au secteur villageois. 59 p. FIRCA, Côted'Ivoire.

3. Barro-Kondombo, C., Sagnard, F., Chantereau, J., Deu, M., Vom Brocke, K, Durand, P., Gozé, E., Zongo, J.D. (2010). Genetic structure among Sorghum landraces as revealed by morphological 
variation and microsatellites markers in three agroclimatic regions of Burkina Faso. Theor. Appl. Genet., 10 (120): 1511-1523.

4. Bazile, D., \& Abrami, G. (2008). Des modèles pour analyser ensemble les dynamiques variétales du sorgho dans un village malien. Cahiers Agricultures, 17 (2) : 203-209.

5. Beninga, M. B. (2007). Génétique, amélioration et vulgarisation $d u$ mil : Pennisetum glaucum (L) R. Br. (Poaceae), en Côte d'Ivoire. Thèse de Doctorat d'Etat, 178 p. UFR Biosciences, Université de Cocody, Côte d'Ivoire

6. CIC (2016). Rapport Marché des céréales. Conseil International des Céréales (CIC), GMR p.469-25. http://www.igc.int/downloads/gmrsummary/gmrsummf.pdf.

7. Coulibaly, H., Bazile, D., Sidibé, A., \& Abrami, G. (2008). Les systèmes d'approvisionnement en semences de mils et sorghos au Mali : production, diffusion et conservation des variétés en milieu paysan. Cahiers Agricultures, 17 (2) : 199-202.

8. Dansi, A., Adoukonou-Sagbadja, H., \& Vodouhè, R. (2010). Diversity, conservation and related wild species of Fonio millet (Digitaria spp.) in the northwest of Benin. Genet. Resour. Crop Evol., 9 (57): 827-839.

9. DGPE (2015). Les politiques agricoles a travers le monde quelques exemples principales données. Côte d'ivoire. Direction Générale de la Performance Economique et Environnementale des Entreprises (DGPE), France, 9 pp. http://agriculture.gouv.fr/politiques-agricolesfiches-pays

10. Djeni, T. (2009). Typologie de l'attiéké de trois zones de production de Côte d'Ivoire et analyse des propriétés des levains traditionnels utilisés pour sa préparation. 206 p. Thèse de Doctorat, Université d'Abobo Adjamé, Côte d'Ivoire.

11. FAO (2009). Traité international sur les ressources phytogénétiques pour l'alimentation et l'agriculture. FAO, 42 p.

12. OUA (2002). Projet Modèle Loi Nationale sur la Sécurité en Biotechnologie. OUA, 44 p.

13. Missihoun, A. A., Agbangla, C., Adoukonou-Sagbadja, H., Ahanhanzo, C., \& Vodouhe R., (2012). Gestion traditionnelle et statut des ressources génétiques du sorgho (Sorghum bicolor L. Moench) au Nord-Ouest du Bénin. Int. J. Biol. Chem. Sci. 6 (3): pp.1003-1018.

14. Sangaré, A., Koffi, E., Akamou, F., \& Fall M. A. (2009). Rapport national sur l'état des ressources phytogénétiques pour l'alimentation et l'agriculture, $65 \mathrm{p}$. 
15. Silue, N., Fofana, I.J., Silue, S., Diarrassouba, N., Kouassi, A. F., \& Kouakou K. (2014). Identification des espèces ligneuses utilisées dans l'alimentation des bovins dans la région du Poro (Nord de la Côte d’Ivoire). Agronomie Africaine 2 (6): 217229. 